Relations industrielles

Industrial Relations

\title{
Organizational Behavior, by Joe Kelly, Richard D. Irwin Inc., Georgetown, Ontario, 1969, 666 pp.
}

\section{Laurent Bélanger}

Volume 24, numéro 4, 1969

URI : https://id.erudit.org/iderudit/028086ar

DOI : https://doi.org/10.7202/028086ar

Aller au sommaire du numéro

Éditeur(s)

Département des relations industrielles de l'Université Laval

ISSN

0034-379X (imprimé)

1703-8138 (numérique)

Découvrir la revue

Citer ce compte rendu

Bélanger, L. (1969). Compte rendu de [Organizational Behavior, by Joe Kelly, Richard D. Irwin Inc., Georgetown, Ontario, 1969, 666 pp.] Relations

industrielles / Industrial Relations, 24(4), 812-813.

https://doi.org/10.7202/028086ar

Tous droits réservés (C) Département des relations industrielles de l'Université Laval, 1969
Ce document est protégé par la loi sur le droit d'auteur. L’utilisation des services d'Érudit (y compris la reproduction) est assujettie à sa politique d'utilisation que vous pouvez consulter en ligne.

https://apropos.erudit.org/fr/usagers/politique-dutilisation/ 
Personnel and Industrial Relations : A Managerial Approach, by John B. Miner, Macmillan, New York, 1969, $562 \mathrm{pp}$.

On assiste depuis quelques années à la prolifération d'ouvrages en administration du personnel. Le livre de Miner présente certains aspects originaux. Ainsi, l'accent est mis sur l'entreprise, plutôt que sur l'individu, en prenant l'approche administrative et systématique (input; output).

De plus, l'auteur rappelle les contextes industriels, sociaux, légaux et culturels dans lesquels le directeur du personnel doit agir (Chapitres 3 à 6). Ensuite, Miner fait souvent appel aux recherches pertinentes dans les sciences du comportement. Enfin, l'ouvrage se termine par un modèle intéressant de la fonction «Personnel 》, en se référant aux théories organisationnelles récentes.

Il convient toutefois de mentionner quelques lacunes. Ainsi, un seul chapitre est consacré à la négociation collective et au syndicalisme; c'est nettement insuffisant. Egalement, l'ouvrage aurait pu contenir des études de cas, pour inciter le lecteur à intégrer les connaissances acquises en proposant des solutions réalistes.

Bref, le livre de Miner est d'un réel intérêt pour les étudiants et les praticiens en administration des ressources humaines.

\section{Jean LADOUCEUR}

\section{Organizational Behavior, by Joe Kelly,} Richard D. Irwin Inc., Georgetown, Ontario, 1969, 666 pp.

C'est un des rares volumes écrits par un canadien sur le comportement des individus au sein des grandes organisations. Cependant, comme ce n'est pas un des rares volumes sur le sujet, l'auteur se doit d'être original au moins dans la présentation. Celle-ci, malheureusement, est assez décevante. La plupart des chapitres qui tentent de regrouper une multitude de thèmes n'ont pas de liens serrés entre eux. Le premier qui porte le même titre que le volume essaie de circonscrire le domaine des sciences du comportement et discute de leur utilisation en administration des entreprises. Pour il- lustrer le développement de la théorie des organisations, l'auteur se sert des changements dans le contenu de la tâche de l'administrateur du personnel.

Le thème des relations humaines est repris pour être développé plus longuement dans un deuxième chapitre. On présente, encore une fois, les débuts de la sociologie industrielle et de la psychosociologie de l'entreprise. L'auteur en profite pour insérer les idées de Douglas McGregor (Théorie $\mathrm{X}$ et théorie $\mathrm{Y}$ ) qu'il présente comme deux approches en relations humaines.

Un volume sur le comportement organisationnel doit forcément traiter du leadership. C'est le sujet du troisième chapitre. Après avoir présenté et discuté les deux approches dans l'étude du leadership, l'auteur rapporte les travaux de Fieldler, un auteur peu connu du Québec pour le moment. Fieldler a largement contribué à l'élaboration de la théorie de la relativité des styles de commandement.

Une deuxième section traite des interrelations entre la personnalité, les groupes et l'organisation. C'est une reprise du débat sur l'incompatibilité entre le développement normal de l'individu et les exigences de l'organisation. C'est aussi une présentation des théories sur les structures et le fonctionnement des groupes. Un sixième chapitre vient clore cette section en présentant un cadre de référence propre à l'étude du comportement au sein des organisations. Pour un esprit cartésien, ce chapitre pourrait servir d'introduction.

Avec la troisième section, l'auteur passe aux applications pratiques. Il rapporte une série d'études descriptives touchant le comportement d'un directeurgénéral, d'un gérant de départernent. Ces études constituent les chapitres VII et VIII. Le chapitre IX sur le recrutement et la sélection des dirigeants est remarquable par le traitement qu'il accorde à l'entrevue de placement et à l'utilisation des textes. Cette section se termine avec un chapitre sur les communications, précédé par un chapitre sur la sensibilisation aux relations interpersonnelles.

$\mathrm{Au}$ chapitre XII, on revient à un sujet plus théorique et souvent négligé dans les ouvrages de ce genre: c'est le thème du conflit au sein des organisations: conflits interpersonnels, conflits interdépar- 
tementaux, conflits syndicats-direction. On y décrit les mécanismes de solution des conflits; celui de la théorie des jeux en particulier.

Avec le chapitre XIII, on retourne au domaine des applications en discutant des principes, des méthodes et de l'efficacité des programmes de formation pour dirigeants. En conclusion, l'auteur traite de l'entreprise de l'avenir et fait largement usage des thèses de Galbraith, de Likert, de Stalker et Burns.

En touchant ainsi une foule de thèmes, un auteur peut difficilement échapper au traitement superficiel d'un sujet. Pour parer à cette difficulté et pour apporter plus d'information, l'auteur insère dans un rectangle un résumé d'articles intéressants publiés dans des revues spécialisées.

L'auteur se proposait de donner une vue 《compréhensive» de la masse d'information dont on dispose actuellement sur les déterminants structurels et personnels du comportement organisationnel. Il voulait aussi mettre de l'ordre dans cet amas de connaissances. Nous croyons que l'auteur a atteint son premier objectif mais non le second.

\section{Laurent BELANGER}

Emerging Concepts in Management, by Max Wortman and Fred Luthans, Macmillan, New York, 1969, 462 pp.

Ce livre contient une sélection de 46 articles publiés depuis moins de cinq années. Ces articles sont représentatifs des récents développements dans la littérature administrative.

La première partie analyse les fondements théoriques et sociaux de l'administration. On y rappelle les difficultés d'unifier les multiples philosophies et théories publiées jusqu'à ce jour. La seconde partie analyse les fonctions administratives: planification, organisation, direction, contrôle. La contribution des sciences sociales est le sujet de la troisième partie; on $y$ traite des recherches récentes en comportement et changement organisationnel, en motivation et intégration personnelle.

Les sections IV et $\mathrm{V}$ de l'ouvrage sont consacrées à l'approche quantitative (re- cherche opérationnelle) et à l'analyse systématique en prenant l'organisation comme cadre de référence. $\mathrm{La}$ dernière partie présente des perspectives nouvelles sur l'administration multinationale et l'avenir des organisations.

Il convient d'indiquer quelques particularités. Chaque section est précédée d'une introduction et est suivie de questions à discuter et de lectures supplémentaires recommandées. Les articles ont été sélectionnés pour donner une vue d'ensemble des théories administratives et des tendances futures. Leurs auteurs appartiennent à plusieurs Ecoles de pensée, de sorte que le lecteur est stimulé à faire un jugement personnel sur les opinions exprimées.

Cet ouvrage est recommandé à quiconque veut mettre à jour ses connaissances sur les théories et concepts administratifs.

Jean LADOUCEUR

\section{Managerial Process and Organizational} Behavior, by Alan C. Filley \& Robert J. House,-Scott, Foresman and Company ; Glenview, Illinois, 1969, $500 \mathrm{pp}$.

This excellent book integrates recent theories in social sciences and classical management theory. The objective of the authors was to bring together and make operational the relevant findings from research in the litterature of sociology, psychology, political science, economics and business management. The topics are presented in an original way, much different from classical textbooks.

The book is devided into three parts. The first presents information on management thought, the fundamentals of research strategy, and authority structures. The second part deals with particular issues in organization theory : division of labor, organizational and personal objectives, planning, decisionmaking, role conflict, etc. The framework used is «theory - propositions evaluation »; the theory surrounding each issue is explained and resumed in key propositions, then evaluated by research findings. The third part treats leadership, motivation and organizational development. Chapter 18 gives a new 